\title{
Energy efficient routing in wireless sensor network based on mobile sink guided by stochastic hill climbing
}

\author{
Raghavendra Y. M. ${ }^{1}$, U. B. Mahadevaswamy ${ }^{2}$ \\ ${ }^{1}$ Department of Electronics and Communication Engineering, \\ GSSS Institute of Engineering and Technology for Women, Visvesvaraya Technological University, India \\ ${ }^{2}$ Department of Electronics and Communication Engineering, Sri Jayachamarajendra College of Engineering, \\ JSS Science and Technology University, India
}

\begin{tabular}{l}
\hline \hline Article Info \\
\hline Article history: \\
Received Nov 27, 2019 \\
Revised Apr 29, 2020 \\
Accepted May 12, 2020 \\
\hline
\end{tabular}

\section{Keywords:}

Fuzzy

Hill climbing

Mobile sink

Optimization

Wireless sensor network

\begin{abstract}
In wireless sensor networks (WSNs), the minimization of usage of energy in the sensor nodes is a key task. Three salient functions are performed by WSNs' sensor nodes namely data sensing, transmitting and relaying. Routing technique is one of the methods to enhance the sensor nodes battery lifetime. Energy optimization is done by using one of the heuristic routing methods for data sensing and transmission. To enhance the energy optimization mainly concentrated on data relaying. In this work stochastic hill climbing is adapted. The proposed solution for data relaying utilizes geographical routing and mobile sink technique. The sink collects the data from cluster heads and movement of the sink is routed by stochastic hill climbing. Experimentation is done on the network simulator 2 Platform. The existing routing techniques like threshold sensitive energy efficient sensor network, energy-efficient low duty cycle, and adaptive clustering protocol are compared with the obtained results of chosen algorithm. The proposed work shows promising results with respect to lifetime, average energy of nodes and packet delivery ratio.
\end{abstract}

Copyright () 2020 Institute of Advanced Engineering and Science. All rights reserved.

\section{Corresponding Author:}

Raghavendra Y. M.,

Department of Electronics and Communication Engineering,

GSSS Institute of Engineering and Technology for Women, Mysuru,

Visvesvaraya Technological University, Belagavi, Karnataka, India

Email: raghavendra@gsss.edu.in

\section{INTRODUCTION}

In day to day life the WSNs have included a wide range of applications in different types of sectors such as agriculture, tsunami detection, environment and habitat monitoring, ocean boarding alarming for fisherman, traffic control and home automation. The ability of collecting the data forwarding [1] is included in WSNs. The nodes with large number are contained in a typical WSN and distributed around them through the wireless connection. Some environment parameters are sensed by these nodes and these parameters are sent to BS for realization of application loc. The sensors are energized by batteries and as sensors sense, they transmit and receive through multi hop relays and hence the battery energy reduces. After certain point of time sensor nodes fail to function owing to low energy or even battery dead. In most of the cases sensor nodes are primarily positioned in unattended environment and the process may be expensive or may not be impossible for frequent replacement of sensor nodes. This is required to make use of energy prudently in the sensor nodes for improving the network's lifetime. The $[2,3]$ challenges in this field are enormous which include finding the required data, monitoring, compiling, computing, evaluating, and decision-making. WSN starts its function by sensing the data. Different types of sensors are included in the sensing component and the parameters are sensed by sensors such as humidity, temperature, and moisture, etc. relying on the application logic. To handle the data from other devices, transmitters and receivers are contained in the communication component. The battery is contained in the power component that supplies energy to remaining components. 
The programmable microcontroller is included in control components and uses to run the code for controlling the communication components and the sensors.

On the transmission rate, sensing rate, and relay times, the sensor nodes' lifetime or longevity is depended. To reduce energy consumption in data relaying geographical routing solution guided by relay score is proposed. Geographical routing ensures to find shortest path for broadcasting of data to the sink. But the relay times could be even more reduced with usage of mobile sink. In this work mobile sink technique is used which further reduces data relay rate compared to earlier research work. Mobile sink moves around the sensor area and collect packets. The mobile sink movement is guided by an iterative local search algorithm i.e. Stochastic Hill Climbing is used. But in most of the works on mobile sink, movement of mobile sink is fixed and sensors have to adapt their routing based on mobile sink movement. In this work, deviation is made from this approach are based on sensor sensing rate, transmission rate the mobile sink movement is controlled to reduce the relay times.

The manuscript is ordered as follows: In section 2, relevant work is described. Problem definition along with proposed solution is explained in section 3. Performance analysis is discussed in section 4. The simulation results are illustrated in Section 5. In section 6, the conclusion of paper is described finally.

\section{RELATED WORKS}

In [4], the author has been proposed a new protocol known as Equalized Cluster Head Election Routing Protocol (ECHERP). By using the Guassian elimination algorithm, the modeling of a network is considered as a linear system by ECHERP. For improving the lifetime of a network, the nodes' combination which can be selected as cluster head are calculated by this protocol. In multi hops WSNs, an optimization model is presented in [5] for management of a network. For the backup nodes and routes, the algorithm doesn't have been applied as the network is in the static condition but it is left out for saving the energy based on $\mathrm{ON}-\mathrm{OFF}$ cycles. More energy can be saved by these cycles that are taking more time for reactivation of the path to the target than the existing algorithms that can remain the aliving of alternative paths.

In [6], a protocol of equally-weighted multiple least loaded path routing (EMLR) is demonstrated. For promoting the self-adaptability to the dynamics of a network, a localized routing is employed by EMLR for achieving the scalability. By using energy-aware aggregated metric, energy efficiency is achieved. In [7], an algorithm of a new link aware and energy efficient routing for selection of next hop node with efficiency for the transmission of packets. Two groups are divided in the packet based on the proposed approach that involves low and high priority packets. A node is selected that has the least amount of delay to the next node and the hop counts with lowest number to the sink for high priority packets. Whereas for the packets with low preference, a node is chosen that includes the lowest path loss and is nearer to the sink.

In [8], the author was proposed a routing scheme of Integrated Location Service and Routing (ILSR) with the use of geographic routing protocol. Before or after a link breakage and when a link is created, the location of sink is updated to neighboring sensors. In [9], the issues of maximization of lifetime under the environments of multiple mobile sinks are discussed by the author. Here, a novel heuristic method with three-phases is implemented that contains determination of a high-quality trajectory for each mobile sink, computing the time profile of sojourn at each potential sojourn location, and finding out the actual time of sojourn at each location of sojourn. In [10], an artificial neural network with the use of robust and energy-efficient routing scheme for WSNs known as ELDC is proposed by authors. For making the network adative to any type of environment and more reliable, the training of network on huge data sets that contains almost all scenarios is done in this method.

In [11], a new energy efficient protocol is called as threshold sensitive energy efficient sensor network (TEEN) protocol is introduced by authors for re-activation of networks. For critical applications, TEEN is applied well and it is also implemented with efficiency in terms of response time and energy conservation. In [12], the context of mobile sink is proposed by author for solving the energy-hole problem. In [13], a novel hierarchical approach termed as distributed energy efficient adaptive clustering protocol with data gathering (DEACP) is presented. The distance is utilized that is among the cluster-heads in this method for achieving a WSN with well distribution of suitable clusters of particular sizes. In [14-16], mobile sink-based routing protocol solutions are demonstrated for wireless sensor networks. The load on a fixed set of nodes in a network is reduced by the mobile sink and the load is distributed among various sets of sensor nodes according to its current location. From the regions in which human intervention is impossible, the reliable data collection is ensured by the mobile sink. 


\section{PROBLEM DEFINITION}

The WSN is contained $\mathrm{N}$ nodes in which an initial node with different priority levels $\mathrm{P}$ completely is assigened that have a residual energy of $\mathrm{E}$. The location of deployment's sensitivity is relied by the priority level. According to its energy E and priority P, the designing of nodes of sensor network should be done with the speed of sensing of data in the initial step. Through the multi hop routing process, the sensed data is needed to be sent to the BS. The sensor network's lifetime is demonstrated based on the operational time where the initial node is died in the sensor network. Without disturbing the data collection, the target of the solution is to prolong the lifetime of a network.

\subsection{Proposed solution}

In a sensor network energy consumption is modeled as stated:

$$
E(t)=E^{\text {sens }}(t)+E^{T x}(t)+E^{R x}(t)+E^{A c k T x}(t)+E^{A c k R x}(t)+E^{\text {Idle }}(t)
$$

where the energy utilized is specified as follows:

$E^{\text {sens }}(t)$ for sensing over time $t$

$\mathrm{E}^{\mathrm{Tx}}(\mathrm{t})$ for transmission over time $\mathrm{t}$

$E^{\mathrm{Rx}}(\mathrm{t})$ for reception over time $\mathrm{t}$

$\mathrm{E}^{\text {AckTx }}(\mathrm{t})$ for Ack transmission over time $\mathrm{t}$

$E^{A c k R x}(t)$ for Ack reception over time $t$ and

$E^{\text {Idle }}(t)$ for idle stage.

Energy consumption during sensing and processing is provided as

$$
\mathrm{E}^{\text {sens }}(\mathrm{t})=\mathrm{t} * \mathrm{f}_{\mathrm{s}} * \mathrm{P}_{\mathrm{s}} * \Phi \mathrm{t}_{\mathrm{s}}
$$

where

$\mathrm{f}_{\mathrm{s}}$ is the sampling frequency of the nodes

$\mathrm{P}_{\mathrm{s}}$ is the processing power for sensing nodes

$\Phi \mathrm{t}_{\mathrm{s}}$ is the sampling durationof the nodes

Energy consumption during transmission is given as

$$
\begin{aligned}
& \mathrm{E}^{\mathrm{Tx}}(\mathrm{t})=\operatorname{AvgNF}_{\mathrm{Tx}} * \mathrm{P}_{\mathrm{Tx}} * \Phi \mathrm{t}_{\mathrm{Tx}} \\
& \operatorname{AvgNF}_{\mathrm{Tx}}=\mathrm{NF}_{\mathrm{Tx}} * \frac{1-\left(\left(1-(1-\eta)^{2}\right)^{\mathrm{R}+1}\right.}{1-\left(1-(1-\eta)^{2}\right)} \\
& \mathrm{NF}_{\mathrm{Tx}}=\mathrm{t} * \mathrm{f}_{\mathrm{Tx}} \& \mathrm{t} \leq \mathrm{LT}
\end{aligned}
$$

where

$\operatorname{AvgNF}_{\mathrm{Tx}}$ is the average transmitted frames in numbers

$\mathrm{P}_{\mathrm{Tx}}$ is the transmitting power

$\Phi \mathrm{t}_{\mathrm{Tx}}$ is the transmission duration

$\mathrm{NF}_{\mathrm{Tx}}$ is the total trnasmitted frames in numbers

$\eta$ is the probability of error

$\mathrm{R}$ is the retransmission numbers

Reception energy is followed as

$$
\begin{aligned}
& \mathrm{E}^{\mathrm{Rx}}(\mathrm{t})=\operatorname{AvgNF}_{\mathrm{Rx}} * \mathrm{P}_{\mathrm{Rx}} * \Phi \mathrm{t}_{\mathrm{Rx}} \\
& \operatorname{AvgNF}_{\mathrm{Rx}}=\mathrm{NF}_{\mathrm{Tx}} *(1-\eta) * * \frac{1-\left(\left(1-(1-\eta)^{2}\right)^{\mathrm{R}+1}\right.}{1-\left(1-(1-\eta)^{2}\right)}
\end{aligned}
$$

where

AvgNF $\mathrm{Rx}_{\mathrm{R}}$ is the average number of recieved frames

$\mathrm{P}_{\mathrm{Rx}}$ is the reception power

$\Phi t_{\mathrm{Rx}}$ is the recption duration

Energy efficient routing in wireless sensor network based on mobile sink guided by ... (Raghavendra Y. M.) 
The confirmation message about successful reception of total number of frames is given by using ACK frame. For ACK frame's transmission, conservation of energy is mentioned as below

$$
\mathrm{E}^{\mathrm{AckTx}}(\mathrm{t})=\operatorname{AvgNF}_{\mathrm{Rx}} * \mathrm{P}_{\mathrm{AckTx}} * \Phi \mathrm{t}_{\mathrm{AckTx}}
$$

where

$\operatorname{AvgNF}_{\mathrm{Rx}}$ is the average number of recieved frames

$\mathrm{P}_{\text {AckTx }}$ is the Ack transmission power

$\Phi \mathrm{t}_{\mathrm{AckTx}}$ is the time Duration for Ack transmission

During reception of Ack frame, the energy conservation is stated as follows:

$$
\begin{aligned}
& \mathrm{E}^{\operatorname{AckRx}}(\mathrm{t})=\operatorname{AvgNF}_{\text {AckRx }} * \mathrm{P}_{\text {AckRx }} * \Phi \mathrm{t}_{\text {AckRx }} \\
& \operatorname{AvgNF}_{\text {AckRx }}=\mathrm{NF}_{\mathrm{Tx}} *(1-\eta)^{2} * * \frac{1-\left(\left(1-(1-\eta)^{2}\right)^{\mathrm{R}+1}\right.}{1-\left(1-(1-\eta)^{2}\right)}
\end{aligned}
$$

$\operatorname{AvgNF}_{\mathrm{AckRx}}$ is the average Ack reception in numbers

$\mathrm{P}_{\text {AckRx }}$ is the Ack reception power

$\Phi t_{\text {AckRx }}$ is the time duration for Ack reception

The transmission and sampling frequency with respect to lifetime is measured and plotted as shown in Figure 1 [17]. Based on the results, it is observed that improve in both transmission and sampling frequency causes decrease in lifetime of sensor nodes. In Figure 2, the transmitter power effect on lifetime is illustrated. The lifetime is reduced by increasing in transmission power but not in terms of exponential way. However, the lifetime is affected in an exponential form by changing in the transmission rate and sampling frequency.

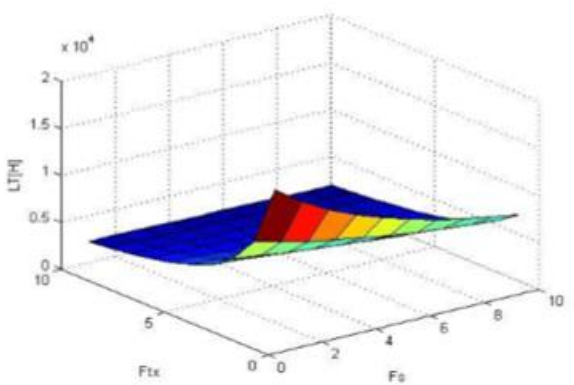

Figure 1. Influence of transmission and sampling on network lifetime

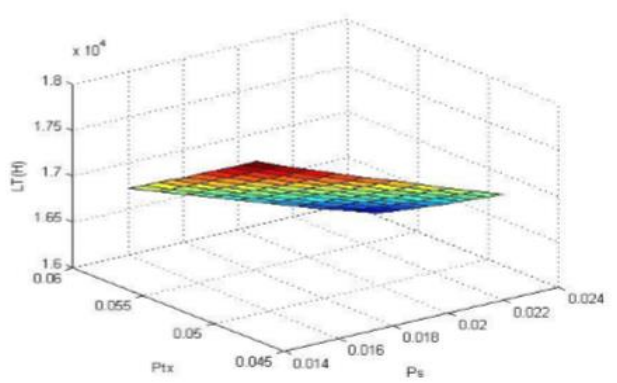

Figure 2. Influence of transmission power on network lifetime

To increase the sensor network's lifetime, the parameters for optimization are described below based on the observation.

- Data sensing rate of node $\left(D_{S}\right)$

- Data transmission rate of node $\left(\mathrm{D}_{\mathrm{T}}\right)$

- Data relay times of node $\left(D_{R}\right)$

In the node's Data sensing rate, sampling frequency is replicated. In the relay rate and data transmission rate, the reflection of transmission rate is done. When these three factors can be reduced, the network's lifetime is improved.

The current work focuses on reducing data relay time. Here Data relay times was reduced using geographical routing based on relay score, still life time is affected when all high priority nodes are on single routing path and geographical routing will not be able to reduce the energy drain. So, a mobile sink is used in the proposed solution to avoid such problems [18]. In the Hill Climbing method, the optimal flow depends up on the number of nodes. The residual energy ' $\mathrm{E}$ ' is increased with the increasing of number of nodes ' $\mathrm{N}$ " and this leads to decreasing the battery performance and the sensors life span. The proposed routing solution is based on guiding the path of sink in such a way to reduce the effective hop count of routing. By reducing the effective hop count, the energy consumption rate is decreased and life time is maximized. 
The proposed mobile sink method finds an optimum position after a time period and then finds an optimum position to move on. The mobile sink calculates the optimum position in such a way to reduce the relay times. In the data packet, hop count and source node information is sent along with information to the sink. The BS is stored the data about the node, hop count and number of times forwarded till the time period. At the end of time period, using this information, calculates the next best position so as to reduce the relay times. Based on the relay score, the routing is performed. A relay score is calculated by each node and a hello packet with the relay score is sent to the area of deployment. The hello packet is received and the sending node's relay score is saved by the neighbor nodes within the area of deployment. The relay score is computed based on the (11):

$$
R_{N}=W 1 * E_{\mathrm{R}}+W 2 * \text { RelayTimes }+W 3 * D_{N B} \quad \text { with }(W 1+W 2+W 3=1)
$$

The equal distribution of proportion of weight is done among distance, relay times, and residual energy [19] such that $(w 1+w 2+w 3)=1$. Where $\mathrm{E}_{\mathrm{R}}$ is the residual energy, Relay times is the number of hops and $\mathrm{D}_{\mathrm{NB}}$ distance of the node from base station [20-22], $(\mathrm{xN}, \mathrm{xB})$ and $(\mathrm{yN}, \mathrm{yB})$ are the node positions. DNB is calculated as

$$
D_{N B}=\sqrt{ }(x N-x B)^{2}+(y N-y B)^{2} / R
$$

$\mathrm{R}$ is communication range of the sensor node.

The sink has to decide the next best position based on the relay hop count of all packets received in the past interval. A cost factor is formulated based on the relay hop count and the best solution which gives higher cost compared to previous solution is chosen for sink movement. This is achieved by using stochastic hill climbing which is a soft computing procedure. All neighbors are evaluated by this algorithm and it chooses the weighted nodes among them based on their evaluation.

\subsection{Stochastic hill climbing}

The overall process of stochastic hill climbing is explained using Pseudo code as shown in the algorithm. Also, pseudo code helps to sink for finding next possible hop to collect data from cluster heads. Cost for the current position is calculated by:

$$
\mathrm{C}=\text { No of packet to sink } / \sum \text { Relay hop Count }
$$

Cost for the new position $(\mathrm{X}, \mathrm{Y})$ is calculated as:

$$
\left.\left.\mathrm{C}=\text { Predicted Packet count to sink / ( } \sum \text { (Distance from }(\mathrm{X}, \mathrm{Y}) \text { to Node Position }\right) / \mathrm{R}\right)
$$

where $\mathrm{R}$ is communication range.

Algorithm: The pseudo-code of the stochastic hill climbing

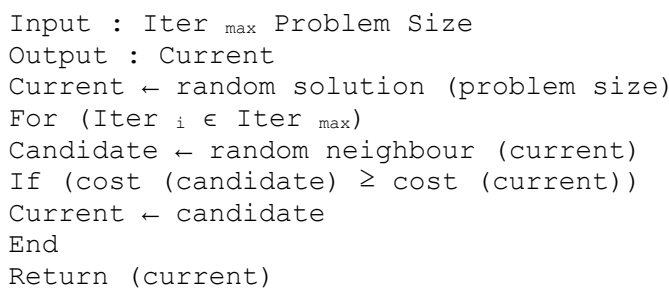

In the discrete domains the Stochastic Hill Climbing with combinational optimization known as explicit neighbors compared to continuous function optimization. The step by step algorithm procedure is applied to continuous domains by the use of neighbor nodes.Even though the technique uses a stochastic process, it can still get stuck in local optima.The Stochastic Hill Climbing will be restarted and repeated for number of times after it convergence to improve the results [23-25].This procedure can be applied to multiple nodes concurrently, allowing multiple algorithm runs to be performed at the same time. Past history of packet count over time period is collected and based on its autoregressive integrated moving average (ARIMA) model is constructed. Due to stochastic hill climbing, the base station converges at optimum position where relay hop count is reduced.

Energy efficient routing in wireless sensor network based on mobile sink guided by ... (Raghavendra Y. M.) 


\section{PERFORMANCE ANALYSIS}

In the proposed scheme, the modeling of life time $(\mathrm{T})$ is done. In the network, the lifetime $(\mathrm{T})$ is the time for energy consumption $\mathrm{E}$ of any node $\mathrm{N}$. The lifetime $(\mathrm{T})$ is not directly proportional to the Data transmission rate $\left(D_{T}\right)$, Data sensing rate $\left(D_{s}\right)$, and the Data relay rate of nodes $\left(D_{R}\right)$.

$$
\begin{aligned}
& \mathrm{D}_{\mathrm{S}} \alpha \frac{1}{T} \\
& \mathrm{D}_{\mathrm{T}} \alpha \frac{1}{T} \\
& \mathrm{D}_{\mathrm{R}} \alpha \frac{1}{T}
\end{aligned}
$$

By combining (13), (14) and (15), the network lifetime (T) can be mentioned as

$$
\left(D_{S} * D_{T} * D_{R}\right)
$$

The Node's residual energy $\left(E_{R}\right)$ is directly proportional to the lifetime.

$$
T \alpha E_{R}
$$

Hence $\mathrm{T}$, where $\mathrm{k}$ is a constant for removing of the proportionality in (19).

$$
\begin{aligned}
& \left(E_{R} / D_{S} * D_{T} * D_{R}\right) \\
& T=k *\left(E_{R} / D_{S} * D_{T} * D_{R}\right)
\end{aligned}
$$

Since $D S \& D T$ are the fuzzy functions.

$$
T=k * \frac{E_{R}}{\frac{\int \mu_{D}^{-}(x) \cdot x d x}{\int \mu_{D}^{-}(x) \cdot d x} * \frac{\int \mu_{D t}^{-}(y) \cdot x d x}{\int \mu_{D}^{-} t^{(y) \cdot d x}} * D_{R}}
$$

With the average length of the routem when routing is happened NR time as,

$$
\begin{aligned}
D R & =k 2 * N R * L A v g \\
T & =\frac{k E_{R}}{\frac{\int \mu_{D r}^{-(x) \cdot x d x}}{\int \mu_{D r}^{-(x) \cdot d x}} * \frac{\int \mu_{D t}^{-}(y) \cdot x d x}{\int \mu_{D t}^{-(y) \cdot d x}} * k 2 * N_{R} * L_{A v g}}
\end{aligned}
$$

If there are high correlation in data values and few high priority nodes, the lifetime is increased in the solution. If the transmission rate is reduced owing to high data correlation and few high priority nodes, the relay times of node is relied on the node's transmission rate. In the proposed solution, the lifetime is improved.

\section{RESULTS}

Through NS2, the simulation of proposed solution was done. Based on the mentioned parameters as shown in Table 1 , the simulation was completed and conducted.

Table 1. Simulation parameters

\begin{tabular}{ll}
\hline Parameters & Values \\
\hline Number of nodes & 50 to 250 \\
Communication range & $100 \mathrm{~m}$ \\
Area of simulation & $1000 \mathrm{~m} * 1000 \mathrm{~m}$ \\
Priority distribution & Uniform distribution with $20 \%$ distribution for each priority \\
Node deployment topology & Random \\
Simulation time & 30 minutes \\
Interface queue length & 50 \\
MAC & 802.11 \\
Number of base station & 1 \\
Location of base station & Upper right \\
Initial energy of nodes & 100 joules \\
Weights W1, W2, W3 & W1=0.2, W2=0.3, W3=0.5 \\
\hline
\end{tabular}


In WSN, the comparison of proposed solution with the routing protocol of TEEN for improved efficiency is mentioned in [11], the solution of ELDC is presented in [10], and a protocol of distributed energy efficient adaptive clustering with data collection for large-scale WSN provided in [13]. The below-mentioned metrics are measured: 1) life time, 2) packet delay, 3) delivery ratio, 4) energy histogram.

Life time is defined as the first sensor node's time or cluster of sensor nodes in the WSN that runs out of energy. It is also described as the maximum duration of time during which the sensors are deployed that have the monitoring capability. As shown in Figure 3, the lifetime is plotted and is measured for various nodes. When compared to the Adaptive clustering protocol [13], TEEN [10], and ELDC [11], the lifetime is improved in the proposed solution. In this work, the consumption of energy by sensor nodes for every round is much less than the other existing methods mentioned in $[10,11,13]$. By comparing with the existing protocol techniques such as provided in $[10,11,13]$, the proposed solution is included the less energy conservation based on the results. The deploying of live nodes' density is done in the area of sensing (D), the available residual energy at the node $(\mathrm{E})$, and the priority of node $(\mathrm{P})$ are affected the data sensing rate. However, the dependency of data transmission rate is on the node's residual energy and the data correlation of sensed value (CT). From the BS, the distance of a node and its energy are impacted the node's data relay rate. Other main reason, for less energy consumption is data relay. Data relay rate in this work is further reduced by using mobile sink guided by stochastic hill climbing search algorithm.

As shown in Figure 4, the packet delay is computed for various nodes. Here the packet delay is lower in the proposed algorithm by comparing with the existing protocol techniques such as $[10,11,13]$. The decrease in packet delay time increases the network lifetime.

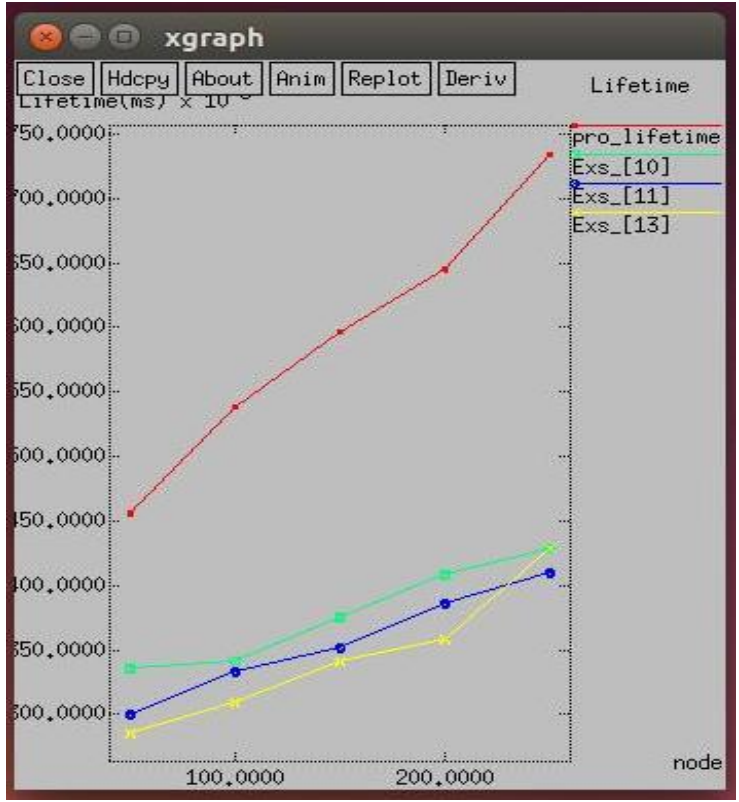

Figure 3. Lifetime versus number of nodes

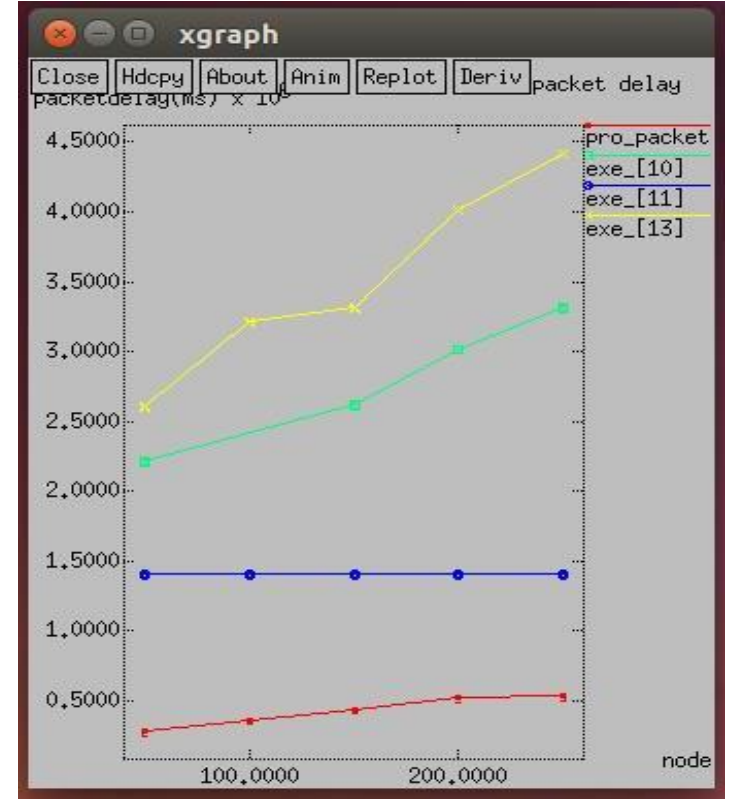

Figure 4. Packet delay versus number of nodes

Packet delay is the propagation time required to deliver packets from node to sink.

Packet delay $=$ Distance/speed of transmission.

Here packet delay is reduced due to reduction in relay rate and data transmission rate. Transmission rate is reduced using fuzzy logic and relay rate is reduced using mobile sink guided by stochastic hill climbing local search algorithm. The capability of protocol in delivering the data packets to the destination is measured by the parameter known as Packet delivery ratio (PDR). PDR is depicted that the ratio of the delivered data packets to the destination node to total transmitted packets.

$\mathrm{PDR}=$ Total Packets received/Total packets transmitted.

Here the packet delivery ratio is enhanced in the proposed algorithm when compared to theexisting protocol techniques such as $[10,11,13]$. In this work, packet delivery ratio is increased because of reduction in data relay rate. Data relay rate is decreased using geographical routing and mobile sink guided by stochastic hill climbing local search algorithm as shown in Figure 5. For the sensor network, all nodes' average energy is computed at time slices with different instances. Figure 6 shows the simulation of nodes' average energy for 
30 minutes in the WSN. The nodes' average value of energy in the evaluated method is higher than the existing techniques like $[10,11,13]$. Because of the decrement in the transmitted number of packets, this improvement is done.

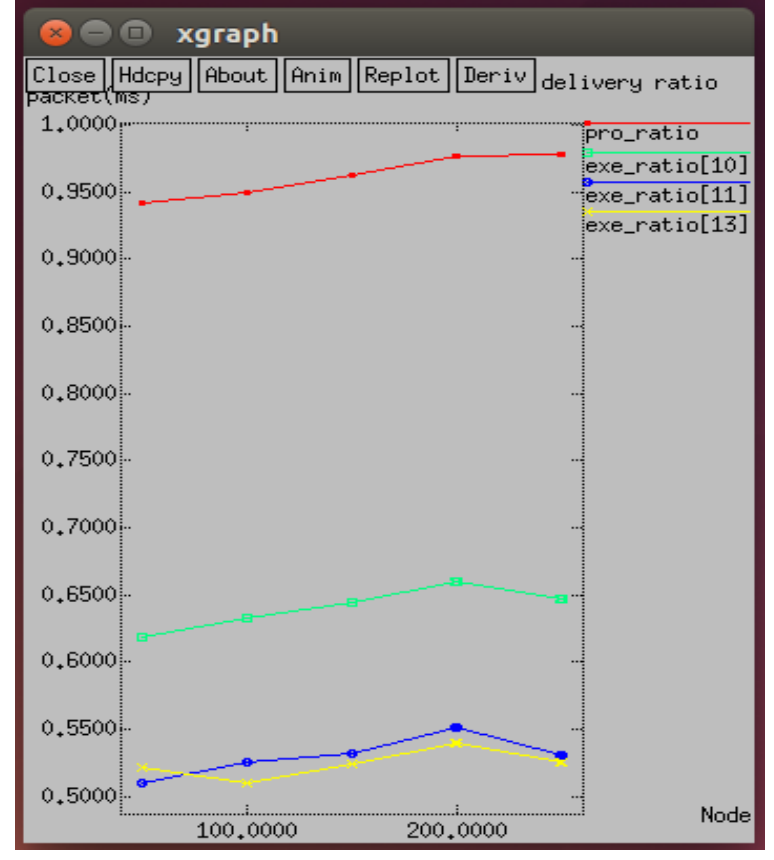

Figure 5. Packet delivery ratio

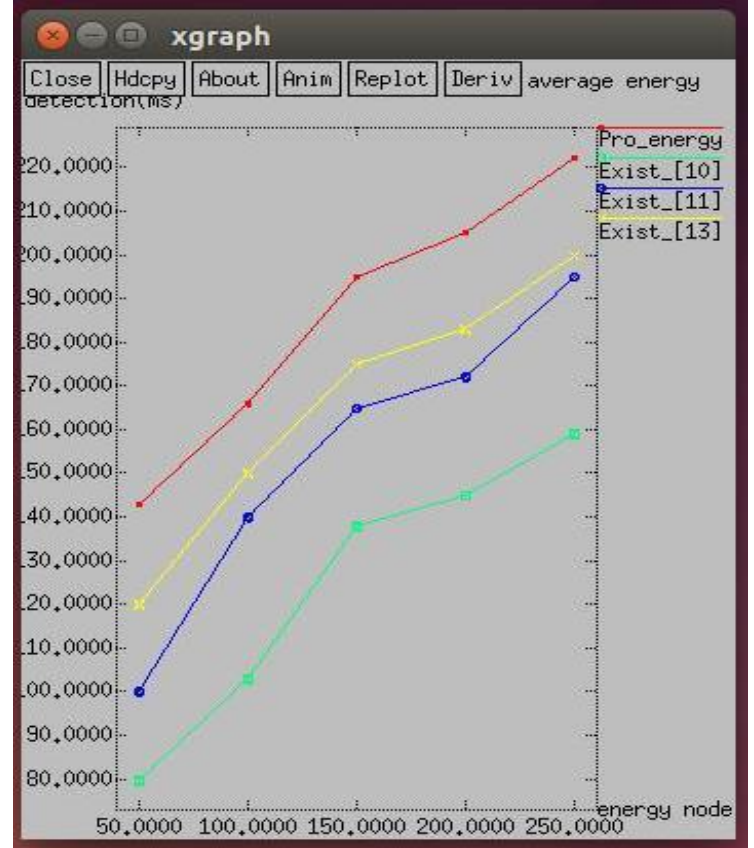

Figure 6. Average energy of nodes

\section{CONCLUSION}

In this work, mobile sink solution guided by stochastic hill climbing is proposed. For optimization of relay hop count; the work proposed mobile sink where movements of mobile sink is guided using stochastic hill climbing. From node to mobile sink geographic routing is applied. For different network configuration, the proposed algorithm was simulated and made a comparision with the existing techniques. The improved lifetime was observed than the other methods. To determine the best configuration of WSN dynamically within the allowed time, a novel method is described in this paper that will improve the target application's performance. On different types of scenarios, the algorithm was run due to that different cases may have unique reward equations.

\section{ACKNOWLEDGEMENTS}

This research was supported in part by SJCE, Mysore, India.

\section{REFERENCES}

[1] S. Rani, et al., "EEICCP-Energy Efficient Protocol for Wireless Sensor Networks," Wireless Sensor Network, vol. 5, no. 7, pp. 127-136, 2013.

[2] Y. M. Raghavendra and U. B. Mahadevaswamy, "Review of Energy Efficient Routing Protocols in Wireless Sensor Networks," Proceedings of Third International Conference on Current Trends in Engineering, Science and Technology, ICCTEST, vol. 1, pp. 1017-1024, 2017.

[3] Y. M. Raghavendra and U. B. Mahadevaswamy, "Energy Efficient Routing in Wireless Sensor Network Based on Composite Fuzzy Methods," Wireless Personal Communications, 2020.

[4] S. A. Nikolidakis, et al., "Energy Efficient Routing in Wireless Sensor Networks Through Balanced Clustering," Algorithms, vol. 6, no. 1, pp. 29-42, 2013.

[5] C. V. Villada and Y. Donoso, "Multipath Routing Network Management Protocol for Resilient and Energy Efficient Wireless Sensor Networks," Procedia Computer Science, vol. 17, pp. 387-394, 2013.

[6] M. M. Monowar, "An energy-aware multi-constrained localized QoS routing for industrial wireless sensor networks," Adhoc \& Sensor Wireless Networks, vol. 36, no. 1-4, pp. 21-50, 2017. 
[7] H. Khodabandeh, et al., "Link aware and energy efficient routing algorithm in wireless body area networks," Network Protocols and Algorithms, vol. 9, no. 1-2, pp. 126-138, 2017.

[8] X. Li, et al., "Localized Geographic Routing to a Mobile Sink with Guaranteed Delivery in Sensor Networks," IEEE Journal Selected Areas in Communication, vol. 30, no. 9, pp. 1719-1729, 2012.

[9] W. Liang, et al., "A Network Lifetime Maximization in Sensor Networks with Multiple Mobile Sinks," Proceedings of 2011 IEEE 36th Conf. Local Computer Networks, pp. 350-357, 2011.

[10] A. Mehmood, et al., "ELDC: an artificial neural network-based energy-efficient and robust routing scheme for pollution monitoring in WSNs," IEEE Transactions on Emerging Topics in Computing, vol. 8, no. 1, pp. 106-114, 2020.

[11] A. Manjeshwar, et al., "TEEN: A routing protocol for Enhanced Efficiency in Wireless Sensor Networks," in Proceedings of 15th International Parallel and Distributed Processing Symposium (IPDPS'01), pp. 2009-2015, 2001.

[12] A. Mehto, et al., "A review on rendezvous-based data acquisition methods in wireless sensor networks with mobile sinks," Wireless Networks, vol. 26, pp. 2639-2663, 2020.

[13] C. Gherbi, et al., "Distributed energy efficient adaptive clustering protocol with data gathering for large-scale wireless sensor networks," 12th International Conference Programming and Systems (ISPS), pp. 1-7, 2015.

[14] S. Jain, et al., "QWRP: Query-driven virtual wheel-based routing protocol for wireless sensor networks with mobile sink," Journal of Network and Computer Applications, vol. 147, p. 102430, 2019.

[15] S. Jain, et al., "QRRP: AQuery-driven Ring Routing Protocol for Mobile Sink based Wireless Sensor Networks," TENCON 2019 - 2019 IEEE Region 10 Conference (TENCON), Kochi, India, pp. 1986-1991, 2019.

[16] Meera G. S. and Priya S. S., "A Survey on routing protocols for mobile sink based WSN," International Research Journal of Engineering and Technology (IRJET), vol. 03, no. 01, pp. 895-901, 2016.

[17] M. U. Younus, "Analysis of the Impact of Different Parameter Settings on Wireless Sensor Network Lifetime," International Journal of Advanced Computer Science and Applications, vol. 9, no. 3, pp. 16-21, 2018.

[18] S. Tabibi and A. Ghaffari, "Energy Efficient Routing Mechanism for Mobile Sink in Wireless Sensor Networks Using Particle Swarm Optimization Algorithm," Wireless Personal Communications, vol. 104, no. 1, pp. 1-18, 2018.

[19] H. K. D. Sarma, et al., "E2R2: Energy-Efficient and Reliable Routing for Mobile Wireless Sensor Networks," IEEE Systems Journal, vol. 10, no. 2, pp. 604-616, 2016.

[20] E. Ahvar, et al., "An Energy-Aware Routing Protocol for Query-Based Applications in Wireless Sensor Networks The Scientific World Journal, pp. 1-9, 2014.

[21] A. R. Bhangwar, et al., "WETRP: Weight Based Energy \& Temperature Aware Routing Protocol for Wireless Body Sensor Networks," IEEE Access, vol. 7, pp. 87987-87995, 2019.

[22] B. Rajasekaran and C. Arun, "Link Failure Detection and Classification in Wireless Sensor Networks using Classification Method," International Journal of Innovative Technology and Exploring Engineering (IJITEE), vol. 8, no. 12, pp. 1132-1135, 2019.

[23] B. Mondal, et al., "Load Balancing in cloud computing using stochastic hill climbing-A Soft Computing Approach," Procedia Technology, vol. 4, pp. 783-789, 2012.

[24] J. Helkey and L. Holder, "Sensor Network Configuration Learning for Maximizing Application Performance," Sensors, vol. 18, no. 6, p. 1771, 2018.

[25] M. J. Rani, et al., "Hybrid Evolutionary Techniques for Ultra Wide Band Sensor Network Localization," International Journal of Recent Technology and Engineering (IJRTE), vol. 7, no. 5S3, pp. 464-467, 2019.

\section{BIOGRAPHIES OF AUTHORS}

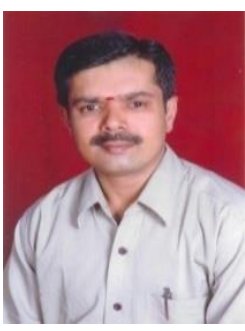

Mr. Raghavendra Y. M., completed his B.E.Degree in Electronics and Communication from Visvesraya Technological University, Belguam in the year 2007, M.Tech in VLSI Design and Embedded Systems from VTU in 2013 and He is presently working as Assistant Professor in the Department of Electronics and communication, GSSSIETW, Mysore, Karnataka, India. He is doing his Ph.D in the area of Wireless Sensor Networks under the guidance of Dr.U.B.Mahadevaswamy in SJCE research centre. His field of interest includes Wireless Sensor Networks, Computer Communication Networks, Embedded Systems and Power Electronics.

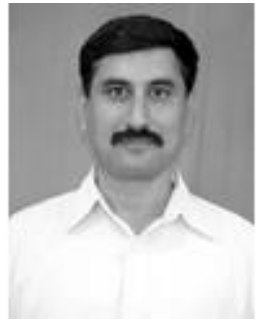

Dr. U. B. Mahadevaswamy completed his B.E. degree in Electronics and Communication from University of Mysore in the year 1988, M.Tech in Industrial Electronics from Mangalore University in the year 1995 and obtained his Ph.D in the field of Wireless Sensor Networks from Mysore University in 2013. He is presently working as Professor in the Department of Electronics and communication, Sri Jayachamarajendra college of Engineering, Mysore, Karnataka, India. He his guiding several research scholars and has published many books and papers both in National and International conferences and Journals. His field of interest includes Wireless sensor networks, Analog and mixed mode VLSI circuits, Control systems, Digital signal processing. 